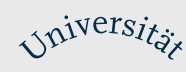

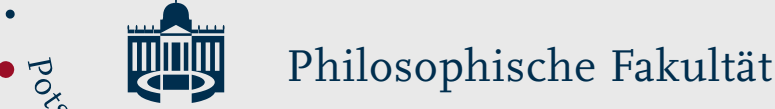

Volkhard Wels

\title{
imaginatio oder inventio
}

Das dichterische Schaffen und sein Gegenstand bei Puttenham, Sidney und Temple

Suggested citation referring to the original publication:

Poetica : Zeitschrift für Sprach- und Literaturwissenschaft 37 (2005) 1/2, S. 65-91 ISSN 0303-4178

Postprint archived at the Institutional Repository of the Potsdam University in:

Postprints der Universität Potsdam

Philosophische Reihe ; 93

ISSN 1866-8380

http://nbn-resolving.de/urn:nbn:de:kobv:517-opus4-87216 

imaginatio oder inventio

Das dichterische Schaffen und sein Gegenstand bei Puttenham, Sidney und Temple 

Volkhard Wels

\section{imaginatio oder inventio}

Das dichterische Schaffen und sein Gegenstand bei

Puttenham, Sidney und Temple

Universität Potsdam 
Dieses Werk ist unter einem Creative Commons Lizenzvertrag lizenziert:

Namensnennung - 4.0 International

Um die Bedingungen der Lizenz einzusehen, folgen Sie bitte dem Hyperlink:

http://creativecommons.org/licenses/by/4.0/

Universität Potsdam 2016

Online veröffentlicht auf dem

Publikationsserver der Universität Potsdam::

URN urn:nbn:de:kobv:517-opus4-87216

http://nbn-resolving.de/urn:nbn:de:kobv:517-opus4-87216 
Volkhard Wels (Potsdam)

IMAGINATIO ODER INVENTIO

Das dichterische Schaffen und sein Gegenstand bei Puttenham, Sidney und Temple

\section{Das Problem}

In Shakespeares A Midsummer Night's Dream (1600) wirft Theseus den Dichtern, den Verrückten und den Liebenden Unglaubwürdigkeit vor, weil alle drei sich nicht von der „,kalten Vernunft“, sondern von der Einbildungskraft (,imagination“) leiten lassen würden:

[...] I never may believe

These antique fables, nor these fairy toys.

Lovers and madmen have such seething brains,

Such shaping fantasies, that apprehend

More than cool reason ever comprehends.

The lunatic, the lover, and the poet

Are of imagination all compact.

One sees more devils than vast hell can hold:

That is the madman. The lover, all as frantic,

Sees Helen's beauty in a brow of Egypt.

The poet's eye, in a fine frenzy rolling,

Doth glance from heaven to earth, from earth to heaven,

And as imagination bodies forth

The forms of things unknown, the poet's pen

Turns them to shapes, and gives to airy nothing

A local habitation and a name. (V.1, v. 2-17) ${ }^{1}$

Den Schöpfungen des Dichters kommt keine Wirklichkeit zu, sie sind ein „luftiges Nichts“, „Formen unbekannter Dinge“, wie etwa die antiken Fabelwesen, denen keine Realität zukommt, auch wenn der Dichter diesen Eindruck erweckt, indem er ihnen Gestalt und einen Namen gibt. Mit solchen Erfindungen kann der Dichter weder Glaubhaftigkeit beanspruchen, noch für sich selbst einen angesehenen Platz in der höfischen Gesellschaft einfordern. Er steht neben dem Verrückten und dem Liebenden, mit denen

\footnotetext{
1 William Shakespeare, A Midsummer Night's Dream, in: The Norton Shakespeare, hg. von Stephen Greenblatt, New York/London 1997, S. 805-863, hier S. 851.
} 
er gemein hat, daß seine Einbildungskraft stärker ist als der „kalte Verstand“.

Dem Dichter wird damit genau das zum Vorwurf gemacht, was Ronsard verhindern wollte, wenn er, fast ein halbes Jahrhundert früher, in seiner Poetik die Erfindungen des Dichters gegen die ,phantastischen und melancholischen“ Erfindungen des Verrückten abzugrenzen versucht: „Quand je te dy que tu inventes choses belles \& grandes, je n'entends toutesfois ces inventions fantasticques \& melencoliques, qui ne se rapportent non plus l'une à l'autre que les songes entrecoupez d'un frenetique, ou de quelque patient extremement tourmenté de la fievre, à l'imagination duquel, pour estre blessée, se representent mille formes monstrueuses sans ordre ny liayson [...]. “2 Ronsard antwortet mit seiner Unterscheidung zwischen den „, choses belles \& grandes“ des Dichters und den ,inventions fantasticques \& melencoliques" des Verrückten oder des Fieberkranken, deren Vorstellungsvermögen ,verletzt“ ist, genau auf den Vorwurf, den der Theseus Shakespeares erhoben hat. Um das dichterische Schaffen gesellschaftlich zu legitimieren, muß ein Kriterium benannt werden, das die Erfindungen des Dichters von den Wahnvorstellungen des Verrückten unterscheidet.

Wie genau das dichterische Schaffen vorzustellen ist, hatte Ronsard schon in dem vorangehenden Satz beschrieben: „L'invention n'est autre chose que le bon naturel d'une imagination concevant les Idées \& formes de toutes choses qui se peuvent imaginer tant celestes que terrestres, animées ou inanimes [sic], pour apres les representer, descrire \& imiter [...].“3 Zweierlei ist hier festzuhalten. Erstens: Die Tätigkeit des Dichters besteht in einer inventio, d.h. in einem rhetorisch-dialektischen Findungsprozess, der aber seinerseits ein gutes Vorstellungsvermögen (,imagination“) voraussetzt, d.h. auf physiologischen und psychologischen Voraussetzungen beruht. Physiologisch und psychologisch sind diese Voraussetzungen insofern, als die imaginatio im Sinne der Fakultätenpsychologie gedacht wird, d. h. die Tätigkeit des Gehirns in mehrere psychologische Fakultäten (sensus communis, imaginatio, ratio, memoria) unterschieden wird, die dann physiologisch in den sogenannten Ventrikeln, den drei Kammern des Gehirns, verortet wurden. Wahnvorstellungen entstehen dieser medizinischen Vorstellung zufolge dadurch, daß die imaginatio z. B. durch eine Kopfverletzung oder durch Fieber beschädigt, der Kontrolle der ratio (,cool reason“ in den Worten Shakespeares) entzogen wird und in der Folge Bilder produziert, die keinen Sinneseindrücken entsprechen, also phantastisch im Sinne

2 Pierre de Ronsard, Abbregé de l'Art poëtique françois, in: ders., Euvres complètes, hg. von Paul Laumonier, Bd. 14, Paris 1949, S. 1-38, hier S. 13.

3 S. $12 \mathrm{f}$. 
des Wortes sind. ${ }^{4}$ Der Vergleich zum dichterischen Schaffen liegt auf der Hand, denn auch dort werden Bilder erzeugt, denen keine Sinneseindrücke entsprechen. In den Worten Shakespeares: der Dichter und der Verrückte ,are of imagination all compact“.

Zweitens ist dieser Ronsard-Stelle zu entnehmen, daß das dichterische Schaffen in einem „Darstellen, Beschreiben und Nachahmen“ der Dinge besteht, die durch die inventio gefunden wurden, nämlich der „Idées \& formes de toutes choses“. Daß die „Ideen“ des Dichters mit den „Formen der Dinge", die er in seiner Dichtung darstellt, identisch sind, überrascht insofern nicht, als der lateinische Begriff der „,forma“ die traditionelle Übersetzung des griechischen ,idea“ darstellt. Was dagegen überrascht, ist die Tatsache, daß der Begriff der „Idee“ im Kontext einer rhetorisch-dialektischen inventio überhaupt auftaucht. Gegenstand der inventio sind nicht „Ideen“, sondern Sachverhalte (,res“) oder Argumente. Es ist insofern höchst bezeichnend, daß Martin Opitz, der ein halbes Jahrhundert später diese Stelle aus dem Abbregé in seine Deutsche Poeterey (1624) übernimmt, genau auf diesen Begriff der Idee (und auf den der imaginatio) verzichtet: „Die erfindung der dinge ist nichts anders als eine sinnreiche faßung aller sachen die wir vns einbilden kônnen/ der Himlischen vnd jrrdischen/ die Leben haben vnd nicht haben/ welche ein Poete jhm zue beschreiben vnd herfür zue bringen vornimpt [...].“5 Opitz übersetzt die Vorstellung Ronsards zurück in das rhetorische Modell, bei dem „Sachen“ oder „Dinge“ (,res“, wie es in der lateinischen Terminologie heißt) durch eine inventio, die sich als begriffliches Verfahren natürlich mittels der ratio vollzieht, gefunden werden. ${ }^{6}$

${ }^{4}$ Stellvertretend verweise ich auf Ruth Leila Anderson, Elizabethan Psychology and Shakespeare's Plays, Iowa City 1927 und Lawrence Babb, The Elizabethan Malady. A Study of Melancholia in English Literature from 1580 to 1642, East Lansing 1951. Zur mittelalterlichen Vorgeschichte vgl. E. Ruth Harvey, The Inward Wits. Psychological Theory in the Middle Ages and the Renaissance, London 1975.

5 Martin Opitz, Buch von der Deutschen Poeterey, in: ders., Buch von der Deutschen Poeterey (1624). Mit dem „Aristarch“ (1617) und den Opitzschen Vorreden zu seinen „Teutschen Poemata“ (1624 und 1625) sowie der Vorrede zu seiner Übersetzung der „Trojanerinnen“ (1625), hg. von. Herbert Jaumann, Stuttgart 2002, S. 5-74, hier S. 26. Diese und ähnliche Exzerpte in der Deutschen Poeterey sind nachgewiesen und ausführlich diskutiert bei Otto Fritsch, Martin Opitzen's Buch von der deutschen Poeterei. Ein kritischer Versuch, Diss. Halle-Wittenberg, Halle 1884; Christian Wilhelm Berghoeffer, Martin Opitz' Buch von der deutschen Poeterei, Frankfurt a. M. 1888; Georg Wenderoth, „Die poetischen Theorien der französischen Plejade in Martin Opitz' deutscher Poeterei“, in: Euphorion 13/1906, S. 445-468.

6 Es entspricht dieser Tatsache, daß Opitz, im Gegensatz zu Ronsard genauso wie zu Puttenham und Sidney, keine Probleme damit hat, den Dichter vom Verrückten zu unterscheiden. Seine Position entspricht in diesem Punkt derjenigen Temples, wie sie unten dargestellt wird. Das tertium comparationis von Dichtern und Verrückten sind 
Noch einmal zurück zum Theseus Shakespeares. Wie dieser dort das Schaffen des Dichters beschreibt, hat nichts mit dem technischen Prozeß der inventio gemein:

The poet's eye, in a fine frenzy rolling,

Doth glance from heaven to earth, from earth to heaven;

And, as imagination bodies forth

The forms of things unknown, the poet's pen

Turns them to shapes and gives to airy nothing

A local habitation and a name.

Shakespeare beschreibt das dichterische Schaffen hier nicht als ein rationaltechnisches Verfahren des Findens von Argumenten, sondern als einen so-

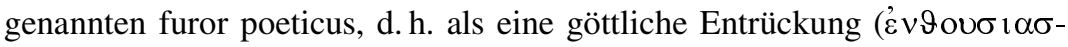
$\mu o ́ s)$, wie sie Platon etwa im Ion (533d-535a) und im Phaidros (245a) entwickelt. Der Dichter wird von einem göttlichen Wahnsinn gepackt und in diesem Zustand der Entrückung einer göttlichen Offenbarung teilhaftig. Aus dieser Tatsache erklärt es sich nach Platon, daß Dichter in ihrem normalen Zustand oft nicht über das Wissen verfügen, das ihre Dichtungen enthalten, ja sogar völlig ungebildete Menschen sein können, die nicht über die Fähigkeiten oder das Wissen verfügen, das ihre Dichtungen voraussetzen. Ebenfalls aus dieser Tatsache erklärt es sich, daß Dichter sich oft nicht mehr erinnern können, wie sie zu ihren Versen gekommen sind, wenn der furor vorübergegangen ist.

Insbesondere Marsilio Ficino hatte Ende des 15. Jahrhunderts in einer ganzen Reihe von Werken diese platonische Theorie des furor poeticus systematisch ausgebaut ${ }^{7}$ und damit erheblichen Einfluß auf die poetologische Entwicklung des 16. Jahrhunderts genommen. Ronsard und die Dichter der Pléiade definieren geradezu ihr Selbstverständnis über Ficinos Enthusiasmus-Theorie. ${ }^{8}$ Auch in England ist diese Theorie rezipiert worden. Neben

die Bilder oder „Ideen“, wenn der Dichter dagegen keine Bilder erschafft, sondern Argumente findet, verliert auch der Vergleich seine Grundlage.

7 Zu Ficinos furor-Theorie verweise ich stellvertretend für die ältere Forschung auf Noel L. Brann, The Debate over the Origin of Genius during the Italian Renaissance. The Theories of Supernatural Frenzy and Natural Melancholy in Accord and in Conflict on the Threshold of the Scientific Revolution, Leiden/Boston/Köln 2002, S. 82-107.

8 Zur Rezeption der furor-Theorie bei Ronsard und der Pléiade vgl. Noel L. Brann, „Melancholy and the divine frenzies in the French Pléiade: their conflicting roles in the art of beaux exercices spirituels", in: The Journal of Medieval and Renaissance Studies 9/1979, S. 81-100; Robert John Clements, „Ronsard and Ficino on the Four Furies“, in: ders./ Robert Valentine Merrill, Platonism in French Renaissance Poetry, New York 1957, S. 118-144; Henri Franchet, Le poète et son æeuvre d'après Ronsard, Paris 1923, Neudr. Genève 1969; Françoise Joukovsky-Micha, Poésie et mythologie 
Shakespeare wäre hier etwa „E. K.“ zu nennen, der anonyme Kommentator von Edmund Spensers Shepheardes Calendar (1579). Er erwähnt den furor poeticus kurz im "Argvment“ der zehnten Ekloge. Cuddie, das vollkommene Vorbild eines Dichters, beschwert sich über die Verachtung und Geringschätzung, die man der Dichtung im elisabethanischen England entgegenbringt, obwohl selbst die barbarischsten Völker sie als eine wertvolle Technik menschlichen Wissens (,arte“) in Ehren gehalten hätten. Aber, so verbessert sich E. K., eigentlich handle es sich bei der Dichtung nicht um eine ,arte“, sondern um eine „,göttliche Gabe“ und einen „,himmlischen Antrieb“, der nicht durch Arbeit und Mühe zu erlangen wäre, sondern durch Enthusiasmus und himmlische Inspiration in den Geist gegossen würde. ${ }^{9}$

Aus dieser Theorie des furor poeticus dürfte es sich erklären, daß der Begriff der imaginatio, der in dem rhetorisch-dialektischen Modell der inventio keine Bedeutung hat, mit der Dichtung überhaupt in Verbindung gebracht wird. Erstens ist es das Vermögen der imaginatio, dem in einer wie auch immer gedachten Entrückung, Offenbarungen' im Sinne des Wortes zuteil werden können. Zweitens ist die imaginatio im Gegensatz zu dem rein begrifflichen Verfahren der inventio ein bildliches Vermögen. Dieses liefert der ratio die bildlichen Vorstellungsinhalte, die allein einem Begriff Konkretheit, Anschaulichkeit und damit Realität verschaffen. Wenn es deshalb bei Puttenham (auf die Stelle wird gleich zurückzukommen sein) heißt, der Dichter wäre Gott zu vergleichen, weil er wie dieser allein aus seiner imaginatio eine Welt hervorbringen könne, so ist dieses „hervorbringen“ im bildlichen Sinne zu verstehen. „Erschaffen“ oder „Schöpfen“ bezieht sich vor allem auf das Vermögen, den Dingen konkrete Anschaulichkeit verleihen zu können. Ein solches Schöpfungsvermögen im emphatischen Sinne,

au XVI siècle. Quelques mythes de l'inspiration chez les poètes de la Renaissance, Paris 1969.

9 Edmund Spenser, The Shepheardes Calender, in: ders., The Works of Edmund Spenser. A Variorum Edition, hg. von Edwin Greenlaw u.a., Bd. 7.1: The Minor Poems, hg. von Charles Grosvenor Osgood und Henry Gibbons Lotspeich, Baltimore 1943, S. 1-120, hier S. 95: „In Cuddie is set out the perfecte paterne of a Poete, which finding no maintenaunce of his state and studies, complayneth of the comtempte of Poetrie, and the causes thereof: Specially hauing bene in all ages, and euen amongst the most barbarous alwayes of singular accounpt and honor, and being indede so worthy and commendable an arte: or rather no arte, but a diuine gift and heauenly instinct not to bee gotten by laboure and learning, but adorned with both: and poured into the witte by a certaine $\varepsilon \vee \vartheta o v \sigma \iota \alpha \sigma \mu$ ó $\varsigma$ and celestiall inspiration [...].“ 
das den äußerst vorteilhaften Vergleich mit Gott erlaubt, läßt sich aus der inventio nicht ableiten.

Damit komme ich zu den Thesen, die ich im folgenden vertreten möchte. Wenn das dichterische Schaffen und sein Gegenstand in der Poetik der englischen Renaissance eine bedeutende Rolle spielt, so ist das vor dem Hintergrund des italienischen Neuplatonismus zu verstehen. Der entscheidende Punkt dabei ist, daß Puttenham und Sidney zwar die platonisch besetzten Begriffe der „Schöpfung“ und der „Idee“ übernehmen, sich ansonsten aber gegenüber dem neuplatonischen Begriff des Dichters und insbesondere des furor poeticus scharf ablehnend verhalten. Hier macht sich eine gewisse Ambivalenz bemerkbar. Einerseits wollen Puttenham und Sidney die Dichtung nicht wieder, wie es das rhetorische Modell impliziert, zu einer bloß schulischen, rein technischen Angelegenheit herabstufen. Das explizite Ziel beider Traktate ist es, die Dichtung als Tätigkeit, die eines ,Gentleman ', eines sich bei Hofe bewegenden Adligen, würdig ist, zu legitimieren. Genau für diese Aufwertung zu einer ,inspirierten', schöpferischen Tätigkeit aber benötigen sie das neuplatonische Modell.

Auf der anderen Seite jedoch gehen ihnen die Implikationen dieses Modells, vor allem, wo sie in die Theologie hinüberreichen, viel zu weit. Ficino hatte den Dichter, über den Enthusiasmus, zu einem Seher und Propheten erklärt und die heidnisch-antike Dichtung als prisca theologia auf den Rang der biblischen Offenbarung erhoben. Daß dies von theologischer Seite als äußerst fragwürdig empfunden wurde, zeigt etwa Girolamo Savonarolas gegen Ficino gerichteter Apologeticus de ratione poeticae artis (1492), in der die Dichtung wieder auf ihren angestammten Platz neben Rhetorik und Dialektik innerhalb des aristotelischen Organon verwiesen wurde. Insbesondere die Reformation hatte sich diese Position zu eigen gemacht und nicht nur der Dichtung jeden prophetischen Charakter bestritten, sondern überhaupt die unmittelbare göttliche Offenbarung, wie sie der Enthusiasmus impliziert, auf das biblische Altertum beschränkt. Dabei kommt erschwerend hinzu, daß die Reformatoren gerade den Begriff des Enthusiasten verwendeten, um radikalprotestantische Denker, wie etwa Karlstadt, Müntzer, Schwenckfeld, Franck oder Weigel als ,Verrückte' auszugrenzen. Auch sie, so lautete das Argument, mäßen ihre scheinbaren Eingebungen oder Offenbarungen nicht an den Forderungen der ratio, sondern ließen sich von ihrer imaginatio verführen. Der dichterischen Berufung auf eine wie auch immer geartete Inspiration war damit auf Jahrhunderte hinaus (noch die pietistischen Sekten wurden als enthusiastisch diffamiert) die theoretische Grundlage und der Zugriff auf die antike Tradition verwehrt.

Dies scheint mir der Rahmen zu sein, innerhalb dessen die Aussagen zum dichterischen Schaffen und seinem Gegenstand in den poetologischen 
Schriften der englischen Renaissance zu verorten sind. ${ }^{10}$ Puttenham und Sidney wollen auf die Begriffe der „Schöpfung“ und der „Idee“, die ihnen eine Aufwertung des dichterischen Schaffens gegenüber einer bloß rhetorisch-technischen Tätigkeit gestattet, nicht verzichten, andererseits aber auch nicht das neuplatonische Modell mit seinen theologischen Implikationen, vor allem was den Enthusiasmus betrifft, übernehmen.

\section{George Puttenhams Arte of English Poesie}

Das erste Kapitel seiner Arte of English Poesie (1589 gedruckt, um 1569 entstanden $)^{11}$ eröffnet George Puttenham mit dem Vergleich von Dichter und Gott, wobei das tertium comparationis die Schöpfung aus dem Nichts mittels der imaginatio ist. ${ }^{12}$,Schöpfung aus dem Nichts', so führt Putten-

10 Dieser Rahmen konnte hier selbstverständlich nur äußerst grob skizziert werden. Der vorliegende Aufsatz stellt einen Ausschnitt aus einer größer angelegten Arbeit des Verfassers zum Begriff der Dichtung in der Frühen Neuzeit dar, auf die für eine umfassende Darstellung des Hintergrundes verwiesen sei.

$11 \mathrm{Zu}$ Puttenham sei stellvertretend verwiesen auf Simeon Kahn Heninger, Touches of Sweet Harmony. Pythagorean Cosmology and Renaissance Poetics, San Marino, Calif. 1974, S. 287-324 und Dorothee Rölli-Alkemper, Höfische Poetik in der englischen Renaissance. George Puttenhams „The Arte of English Poesie“ (1589), München 1996, v. a. S. 37-60. Zum Begriff der imaginatio vgl. besonders die materialreiche Studie von William Rossky, „Imagination in the English Renaissance: Psychology and Poetic“, in: Studies in the Renaissance 5/1958, S. 49-73; außerdem Murray W. Bundy, ,,Invention“ and ,Imagination“ in the Renaissance“, in: The Journal of English and Germanic Philology 29/1930, S. 535-545 und die entsprechenden Kapitel bei Anderson, Elizabethan Psychology (wie Anm. 4). Ausführliche Bibliographie der Forschungsliteratur bei Heinrich Franz Plett, English Renaissance Rhetoric and Poetics. A Systematic Bibliography of Primary and Secondary Sources, Leiden/New York/ Köln 1995.

12 Vgl. George Puttenham, The Arte of English Poesie, hg. von Gladys Doidge Willcock und Alice Walker, Cambridge 1936, Neudr. 1970, S. 3: „A Poet is as much to say as a

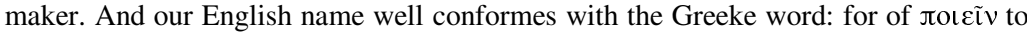
make, they call a maker Poeta. Such as (by way of resemblance and reuerently) we may say of God: who without any trauell to his diuine imagination, made all the world of nought, nor also by any paterne or mould as the Platonicks with their Idees do phantastically suppose. Euen so the very Poet makes and contriues out of his owne braine, both the verse and matter of his poeme, and not by any foreine copie or example, as doth the translator, who therefore may well be sayd a versifier, but not a Poet. [...] And neuerthelesse without any repugnancie at all, a Poet may in some sort be said a follower or imitator, because he can expresse the true and liuely of euery thing is set before him, and which he taketh in hand to describe: and so in that respect is both a maker and a counterfaitor: and Poesie an art not only of making, but also of imitation." 
ham aus, bedeutet, daß der Dichter nicht nach einem Vorbild arbeitet, wie der Übersetzer und bloße Verseschmied (,versifier“), sondern den Stoff und die Form seines Gedichtes selbst erschafft. Ausdrücklich heißt es, daß dieser Schöpfungsprozeß nicht auf so „,phantastische“ Art vorzustellen wäre, wie bei den Platonikern mit ihren Ideen. Obwohl der Dichter zurecht ein Schöpfer genannt wird, kann er genauso auch ein Nachahmer (,,counterfaitor") und die Dichtkunst nicht nur eine Kunst des Machens, sondern auch eine der Nachahmung heißen. Beides ließe sich ohne Widersprüche vermitteln, denn die dichterische Schöpfung bestehe gerade darin, die Sache, die sich der Dichter zu beschreiben vorgenommen hat, getreu und lebendig (d. h. im Sinne der rhetorischen evidentia, der Anschaulichkeit) abzubilden. Das dichterische Schaffen besteht also in der nachahmenden Beschreibung eines bestimmten Sachverhalts, nicht in der ,phantastischen Erfindung ' einer Idee.

Ähnlich zweideutig ist Puttenhams Bestimmung des dichterischen Vermögens. Puttenham listet vier Erklärungsmöglichkeiten auf. Entweder die Dichtkunst geht auf ,einen bestimmten göttlichen Antrieb, die Platoniker nennen es furor“, oder herausragende natürliche Anlagen aufgrund des entsprechenden Temperamentes (,complexion“), oder besonders feine und damit leicht bewegliche spiritus, oder viel Erfahrung und Beobachtung der Welt zurück. ${ }^{13}$ Ziel von Puttenhams Argumentation ist auch hier der Nachweis, daß die Dichter hochachtsame Menschen sind. ${ }^{14}$ Zweierlei fällt jedoch auf. Erstens, daß der furor poeticus von einer Gottbesessenheit zu einem bloßen „divine instinct“ herabgestuft und damit nah an den folgenden Begriff der Begabung herangerückt wird. Zweitens, daß dieser Begriff der Begabung seinerseits medizinisch-physiologisch erklärt wird, indem er einerseits auf das Temperament, andererseits auf die ,spiritus' zurückgeführt wird. Bei diesen ,spiritus' handelt es sich um die ,Lebensgeister', die vom Blut transportiert werden und die materiale Grundlage der Tätigkeit des Gehirns darstellen. Nach den medizinischen Theorien der Zeit geht deren Qualität wesentlich auf das Temperament zurück.

13 Ebd.: „And this science in his perfection, can not grow, but by some diuine instinct, the Platonicks call it furor: or by excellencie of nature and complexion: or by great subtiltie of the spirits \& wit, or by much experience and obseruation of the world, and course of kinde, or peraduenture by all or most part of them."

14 S. 4: „It is therefore of Poets thus to be conceiued, that if they be able to deuise and make all these things of them selues, without any subiect of veritie, that they be (by maner of speech) as creating gods. If they do it by instinct diuine or naturall, then surely much fauoured from aboue. If by their experience, then no doubt very wise men. If by any president or paterne layd before them, then truly the most excellent imitators \& counterfaitors of all others." 
Auch hier geht Puttenhams Bestreben offensichtlich dahin, den platonischen furor poeticus in der Begabung, dem ,instinct diuine or naturall“, aufzulösen. Die zusätzliche Bestimmung dieses ,instinct“ als eines „natürlichen“, also physiologisch im Temperament und der Qualität der ,spiritus“ begründeten, verweist auf ein naturphilosophisches, medizinisches Erklärungsmodell, das die Begabung gerade nicht, wie im Neuplatonismus Ficinos, auf unmittelbar göttliche Einwirkung, sondern auf physiologische Bedingtheiten zurückführt. Dieser Befund bestätigt sich im dritten Kapitel, in dem Puttenham zu begründen versucht, warum die ersten Dichter auch Priester und Propheten waren, und dies dann unter anderem darauf zurückführt, daß die Dichter durch ihre meditative und abstinente Lebensform ihre ,spiritus' so fein und beweglich gemacht hätten, daß sie zum Empfang von Prophetien und Visionen besonders geeignet gewesen wären. ${ }^{15}$

Dieser Befund bestätigt sich schließlich auch im achten Kapitel, wenn Puttenham die Mißachtung beklagt, die man, im Gegensatz zur Antike, am englischen Hof den Dichtern entgegenbringe. Der Dichter, so lautet der Vorwurf, wäre ein Phantastiker (,a phantasticall man“), weil er sich mit

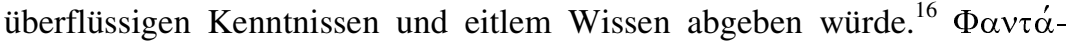
$\sigma \tau \imath \kappa о \varsigma$, so Puttenhams Verteidigung, würde bei den Griechen jemand genannt, bei dem durch eine schlechte Disposition des Gehirns das Urteilsvermögen und die diskursive Vernunft behindert wäre. Wenn der imaginative Teil des Gehirns dagegen geordnet und wohlproportioniert wäre, würde er die Tätigkeit der Vernunft nicht mit monströsen, d. h. unnatürlichen Vorstellungen stören, sondern, ganz im Gegenteil, wie ein Glas oder Spiegel der Seele alle Arten von Anschauungen liefern, ,wodurch dem inventiven Teil des Verstandes so sehr geholfen wird, daß ohne ihn kein Mensch irgend etwas Neues oder Seltenes erdenken könnte." Kein politischer Führer, kein Ingenieur und kein Erfinder könne ohne ein wohlgeordnetes Vorstellungsvermögen irgend etwas in seinem Bereich leisten. Aristoteles und sein Kommentator Alexander von Aphrodisias bestätigten, daß ohne Vorstellungen Denken überhaupt nicht möglich wäre.

Das Vermögen der Phantasie ähnelt einem Spiegel, und wie es Zerrspiegel gibt, die den dargestellten Gegenstand unnatürlich und falsch wiedergeben, so kann eben auch ein schlecht disponiertes, ungeordnetes Vorstellungsvermögen den Gegenstand unnatürlich und falsch wiedergeben und Chimären und Monster hervorbringen. Menschen, deren imaginatio derart gestört ist, sind auch in ihrem alltäglichen Verhalten und Leben gestört und heißen mit Recht Phantastiker. Diejenigen Menschen dagegen, die über ein

\footnotetext{
15 S. 7.

16 S. 18.
} 
besonders wohlgeordnetes Vorstellungsvermögen verfügten, hießen nicht „phantastici“, sondern „euphantasiotae“, ,und mit dieser Art von Phantasie ausgestattet sind alle guten Dichter und bemerkenswerten strategischen Führer, alle klugen Erfinder und Ingenieure, alle politischen Gesetzgeber und politischen Berater, bei deren Tätigkeit der inventive Teil am stärksten in Anspruch genommen wird und für das begründete und richtige Urteilsvermögen des Menschen am wichtigsten ist. “17

Spätestens an dieser Stelle wird also deutlich, daß die imaginatio für Puttenham alles andere als ein selbsttätiges, ,kreatives' Vermögen ist. Puttenhams ganzes Bestreben geht vielmehr dahin, genau dieses selbsttätige ,Schöpfertum“ der dichterischen Phantasie, soweit irgend möglich, zu leugnen. Die imaginatio ist das bildgebende Vorstellungsvermögen, das, wenn es gut entwickelt ist, den ,inventiven Teil“ des Gehirns, nämlich die ratio, unterstützt, indem es deren Begriffe mit bildlichen Vorstellungen versorgt. Wie der Politiker, Ingenieur und Wissenschaftler bedient sich der Dichter

17 S. 18-20: „For as the euill and vicious disposition of the braine hinders the sounde iudgement and discourse of man with busie \& disordered phantasies, for which cause the Greekes call him $\Phi \alpha \nu \tau \alpha \dot{\sigma} \tau \imath \kappa o \zeta$, so is that part being well affected, not onely nothing disorderly or confused with any monstruous imaginations or conceits, but very formall, and in his much multiformitie vniforme, that is well proprotioned, and so passing cleare, that by it as by a glasse or mirrour, are represented vnto the soule all maner of bewtifull visions, whereby the inuentiue parte of the mynde is so much holpen, as without it no man could deuise any new or rare thing: and where it is not excellent in his kind, there could be no politique Captaine, nor any witty enginer or cunning artificer, nor yet any law maker or counsellor of deepe discourse, yea the Prince of Philosophers stickes not to say animam non intelligere absque phantasmate, which text to another purpose Alexander Aphrodiseus well noteth, as learned men know. And this phantasie may be resembled to a glasse as hath bene sayd, whereof there be many tempers and manner of makinges, as the perspectiues doe acknowledge, for some be false glasses and shew thinges otherwise than they be in deede, and others right as they be in deede, neither fairer nor fouler, nor greater nor smaller. There be againe of these glasses that shew thinges exceeding faire and comely, others that shew figures very monstruous \& illfauored. Euen so is the phantasticall part of man (if it be not disordered) a representer of the best, most comely and bewtifull images or apparances of thinges to the soule and according to their very truth. If otherwise, then doth it breede Chimeres \& monsters in mans imaginations, \& not onely in his imaginations, but also in all his ordinarie actions and life which ensues. Wherefore such persons as be illuminated with the brightest irradiations of knowledge and of the veritie and due proprotion of things, they are called by the learned men not phantastici but euphantasiote, and of this sorte of phantasie are all good Poets, notable Captaines stratagematique, all cunning artificers and enginers, all Legislators Polititiens \& Counsellours of estate, in whose exercises the inuentiue part is most employed and is to the sound \& true iudgement of man most needful.“ 
der inventio als einer rationalen Technik, um Argumente zu finden, braucht aber dazu ein funktionierendes - und das heißt, der ratio untergeordnetes Vorstellungsvermögen. Funktioniert das Vorstellungsvermögen dagegen nicht und befreit sich von der Herrschaft der ratio, erzeugt es ,wilde " und ungeregelte Phantasien, wie es bei ,Phantastikern“ und Verrückten zu beobachten ist.

Puttenham unterscheidet also nicht zwischen guten und schlechten Dichtern, ${ }^{18}$ sondern zwischen ,fantastici ' einerseits, d.h. ,Verrückten', bei denen etwa durch Wahnvorstellungen die ratio und das Urteilsvermögen nur noch beschränkt funktionsfähig sind, und ,euphantasiotae ' andererseits, d.h. Menschen, bei denen das Vorstellungsvermögen besonders gut ausgebildet ist und die sich deshalb durch die Tätigkeit ihres Verstandes in den verschiedensten Bereichen auszeichnen. Er widerlegt damit das Argument, das der Theseus des Sommernachtstraums (der im übrigen genau einem solchen Hof vorsteht, an dem Puttenham die Dichtung gepflegt sehen wollte) verwendet, um Dichter als Verrückte zu diffamieren. Wenn Puttenham Psychologie und Dichtkunst in den Begriffen von inventio und imaginatio einander annähert, so tut er dies nicht, um damit die Phantasie als Vermögen aufzuwerten, sondern, genau im Gegenteil, er versucht die Bedrohung, die in dieser Annäherung liegt - Dichter sind durch ihre enthemmte Phantasie den Verrückten ähnlich - zu neutralisieren.

\section{Philip Sidneys Defence of Poetry}

Philip Sidney distanziert sich in schärfster Form vom neuplatonischen furor poeticus und damit etwa auch von dem Modell der Dichtung, wie Ronsard und die Pléiade es in Frankreich entwickelt hatten. In seinem Sonnettzyklus Astrophil and Stella (1591) heißt es, er hätte zwar einiges über den furor poeticus gehört, wisse aber bei Gott nicht, was damit gemeint sei. ${ }^{19}$ Etwas

18 So Rölli-Alkemper, Höfische Poetik in der Renaissance (wie Anm. 11), S. 54. Daß Puttenham mit den Begriffen ,fantastici“ und ,euphantasiotae ",ansatzweise eine Symbiose aus platonischem Gedankengut und Quintilianischer Terminologie“ (ebd. S. 55) vollzieht, ist unwahrscheinlich. Der ,physiologische' Kontext und der Verweis auf Alexander von Aphrodisias (neben Averroes der wichtigste Autor für die entstehende Naturphilosophie) lassen andere Quellen erwarten, keine Poetiker oder Rhetoriker, sondern Naturphilosophen wie etwa Telesio oder Cardano.

19 Philip Sidney, Astrophil and Stella (Sonnet 74), in: ders.: Poems, hg. von William A. Ringler, Oxford 1962, S. 163-237, hier S. 204: „Some do I heare of Poets' furie tell/ But (God wot) wot not what they meane by it [...]" George Chapman zitiert nur wenig später (1614) Ficinos furor-Theorie im Widmungsbrief seiner Homer-Übersetzung, 
weniger polemisch heißt es in der Defence of Poetry (um 1580 entstanden, 1595 gedruckt) angesichts der platonischen Verbannung der Dichter aus dem Staat, daß diesem Urteil ein Selbstwiderspruch zugrundeliege, spreche Platon doch mit dem furor den Dichtern eine weit höhere Erkenntnisquelle zu als er selbst es tue, wenn er die Tätigkeit des Dichtens auf den menschlichen Verstand, also die ratio, beschränke. ${ }^{20}$

Sidneys Ablehnung des furor ${ }^{21}$ und damit jeder prophetischen Kraft spiegelt sich in seiner Einteilung der Dichter. Denn daß es prophetisch inspirierte Dichtung gegeben hat, kann und will Sidney angesichts von David, Salomon und Moses, aber auch (,though in a full wrong divinity“) von Orpheus und Amphion nicht leugnen. Deshalb erklärt er diese Art von ,göttlicher Dichtung“ zur ersten Art. Die zweite Art ist die ,philosophische Dichtung“, d.h. Dichtung mit naturphilosophischen, ethischen oder etwa historischen Inhalten. Da diese Form der Dichtung jedoch nicht „dem Lauf ihrer

vgl. die Nachweise bei Franck L. Schoell, Études sur l'Humanisme continental en Angleterre à la fin de la Renaissance, Paris 1926, Neudr. Genève 1978, S. 4-20.

20 Vgl. Philip Sidney, A Defence of Poetry, hg. von Jan van Dorsten, in: ders.: Miscellaneous Prose of Sir Philip Sidney, hg. von Katherine Duncan-Jones und Jan van Dorsten, Oxford 1973, S. 59-121, hier S. 109. Ich zitiere im folgenden nach dieser Ausgabe. Neben dem Kommentar dieser Ausgabe ist sehr hilfreich die Einführung und der Kommentar von Shepherd: Philip Sidney, An Apology for Poetry or The Defence of Poesy, hg. von Geoffrey Shepherd, London 1965. Aus der umfangreichen Forschungsliteratur verweise ich hier nur auf D.H. Craig, „A Hybrid Crowth, Sidney's Theory of Poetry in An Apology for Poetry", in: English Literary Renaissance 10/1980, S. 183-201; Simeon Kahn Heninger, Sidney and Spenser. The Poet as Maker, University Park, PA/London 1989, S. 223-306; Heninger, Touches of Sweet Harmony (wie Anm. 11), S. 287-324; Forrest G. Robinson, The Shape of Things Known. Sidney's „Apology“ in Its Philosophical Tradition, Cambridge, Mass. 1972 und Phillips Salman, „Instruction and Delight In Medieval and Renaissance Criticism“, in: Renaissance Quarterly 32/1979, S. 303-332. Ausführliche Bibliographie der Ausgaben und Forschungsliteratur bei Plett, English Renaissance Rhetoric and Poetics (wie Anm. 11). Hilfreich beim Verständnis von Sidneys Defence ist auch die Analysis Temples, auf die unten noch ausführlich einzugehen sein wird, vgl. William Temple, Analysis of Sir Philip Sidney's „Apology for Poetry“, hg. und übers. von John Webster, Binghampton, NY 1984. Vgl. dazu auch die Studie des Herausgebers, John Webster, „Oration and Method in Sidney's Apology. A Contem-porary's Account“, in: Modern Philology 79/1981-1982, S. 1-15.

${ }^{21}$ Neben Platon nennt Sidney als Quelle für seine Kenntnis des furor noch Cristoforo Landino, vgl. Sidney, Defence of Poetry (wie Anm. 20), S. 121. Die Referenz muß sich nicht unbedingt auf Landinos Dante-Kommentar beziehen, wie van Dorsten in seinem Kommentar S. 209, darin dem Kommentar von Shepherd S. 236 folgend, vermutet. Landino hat die furor-Theorie in zahlreichen Schriften entwickelt, so in der Einführung zu seiner Vergil-Vorlesung (1462), in der Einleitung zum dritten Buch der Disputationes Camaldulenses (1473 entstanden, 1480 gedruckt) und in seinen Horazund Vergilkommentaren (1482 und 1488). 
eigenen inventio“ („takes not the course of his own invention“) folgt, d.h. keinen fiktiven Charakter hat, läßt Sidney die Frage offen, ob es sich dabei überhaupt um echte Dichtung handelt, und nicht bloß um versifizierte Wissenschaft. Die dritte Art ist demgegenüber die eigentliche Dichtung, weil sie dem genuinen Zweck der Belehrung und Unterhaltung durch Nachahmung entspricht. ${ }^{22}$

Mit dieser Dreiteilung der Dichtung ist das Problem der göttlichen Inspiration beseitigt. Es gibt diese Inspiration, aber sie ist auf das biblische $\mathrm{Al}$ tertum und die Antike beschränkt, die moderne Dichtung dagegen ist eine vernunftgeleitete Tätigkeit, die eine gesellschaftlich wichtige Funktion (prodesse und delectare) erfüllt. Die Dichtung steht innerhalb des Kanons der Wissenschaften und Künste und hat keinen besonderen Zugang zu irgendwelchen Wahrheiten. Soweit ist die Argumentation Sidneys ganz traditionell. Neu ist allerdings die Bestimmung des Unterschiedes zu den anderen Wissenschaften und Künsten. Dort nämlich, wo die anderen Wissenschaften die Natur zum Objekt haben und auf deren Beobachtung und Bearbeitung angewiesen sind, erschaffe allein der Dichter ,lifted up with the vigour of his own invention“, d. h. von der Anschaulichkeit seines Erzählens, der Dynamik seiner Fiktion gepackt, eine ,andere Natur ${ }^{6}{ }^{23}$ Diese andere Natur ist sowohl eine Verbesserung und Vollendung der ersten als auch eine Neuschöpfung, insofern dort Geschöpfe möglich sind, die es in der gegebenen, tatsächlichen Natur nicht gibt (Helden, Halbgötter, Zyklopen und Chimären). Wie die tatsächliche Natur vom Sternenhimmel begrenzt wird, so wird die dichterische Schöpfung vom Verstand und Scharfsinn des Dichters begrenzt. Während deshalb die tatsächliche Natur eine eherne ist, ist die vom Dichter erschaffene Natur eine vollkommenere, eine goldene. ${ }^{24}$

22 Sidney, Defence of Poetry, S. 81, Zitate S. 80. Sidney übernimmt diese Dreiteilung von Iulius Caesar Scaliger, Poetices libri septem. Sieben Bücher über die Dichtkunst, hg. von Luc Deitz und Gregor Vogt-Spira, Stuttgart-Bad Cannstatt, hier Bd. I.2 (1994), S. 84 ff.

$23 \mathrm{Zu}$ dieser Interpretation von „lifted up with the vigour of his own invention“ vgl. Temples Paraphrase mit „in producti opificii expolitione“ (Temple, Analysis [wie Anm. 20], S. 76). Webster hat in seiner Edition der Analysis völlig zurecht darauf hingewiesen, daß Temple den Begriff der „inventio“ auf „die Ausgestaltung des [dichterischen] Werkes" bezieht und also nicht im Sinne eines psychologischen Vermögens deutet, vgl. Temple, Analysis, S. 181, Anm. 22. Gleiches gilt für Sidney. „Vigour“ verweist, wie auch Temples Begriff der „expolitio“, auf das rhetorische Prinzip der evidentia. In diesem Sinne auch Craig, Hybrid Crowth (wie Anm. 20), S. $198 \mathrm{ff}$.

24 Sidney, Defence of Poetry, S. 78: „Only the poet, disdaining to be tied to any such subjection, lifted up with the vigour of his own invention, doth grow in effect another nature, in making things either better than nature bringeth forth, or, quite anew, forms 
Ein ähnliches Rangverhältnis bestünde, was den Menschen selbst und seine dichterische Darstellung betrifft. Denn wo die Natur selbst nur ,gemischte Charaktere' hervorbringe, da gelinge es dem Dichter, vollkommene Menschen $\mathrm{zu}$ schaffen. Kein wirklicher Liebhaber ist so beständig wie Theagenes, kein wirklicher Freund so zuverlässig wie Pylades, kein wirklicher Ritter so tapfer wie Orlando und kein wirklicher Fürst so vorbildlich wie Xenophons Cyrus. Dabei mache es keinen Unterschied, daß die Werke der Natur wesenhaft (,essential“) seien, die Werke des Dichters dagegen auf Nachahmung oder Fiktion (,imitation or fiction“) beruhten, denn die Fähigkeit des Künstlers bestehe nicht in dem Werk selbst, sondern in der Idee oder dem Vorentwurf des Werkes (,idea or fore-conceit of the work"). $\mathrm{Daß}$ der Künstler eine solche Idee habe, sei offensichtlich, denn diese Idee sei es ja, die er so, wie er sie ersonnen habe, in seinem Werk hervorbringe. Dieses Hervorbringen der Idee sei nicht ausschließlich imaginativ (,not wholly imaginative"), d. h. phantastisch und fiktiv im Sinne von Luftschlössern und Wahnideen, sondern substantiell und real. ${ }^{25}$

Die dichterische Idee hat nicht nur einen höheren Realitätsgrad als ein Luftschloß, sondern auch als das tatsächlich vorhandene Werk der Natur. Sidney begründet diese Behauptung an dem Beispiel der Cyrus-Figur Xenophons. Der Cyrus, wie Xenophon ihn darstelle, als das Vorbild und Modell eines Fürsten, sei nicht nur eine partikuläre, einmalige Vollkommenheit, wie sie der Natur zufällig in diesem oder jenem Fürsten gelingen mag, sondern als Vorbild und Modell, d. h. eben als ,Idee', von einem viel höhe-

such as never were in nature, as the Heroes, Demigods, Cyclops, Chimeras, Furies, and such like: so as he goeth hand in hand with nature, not enclosed within the narrow warrant of her gifts, but freely ranging only within the zodiac of his own wit. Nature never set forth the earth in so rich tapestry as divers poets have done; neither with so pleasant rivers, fruitful trees, sweet-smelling flowers, nor whatsoever else may make the too much loved earth more lovely. Her world is brazen, the poets only deliver a golden."

25 S. $78 \mathrm{f}$.: „But let those things alone, and go to man - for whom as the other things are, so it seemeth in him her uttermost cunning is employed - and know whether she have brought forth so true a lover as Theagenes, so constant a friend as Pylades, so valiant a man as Orlando, so right a prince as Xenophon's Cyrus, so excellent a man every way as Virgil's Aeneas. Neither let this be jestingly conceived, because the works of the one be essential, the other in imitation or fiction; for any understanding knoweth the skill of each artificer standeth in that idea or for-conceit of the work, and not in the work itself. And that the poet hath that idea is manifest, by delivering them forth in such excellency as he had imagined them. Which delivering forth also is not wholly imaginative, as we are wont to say by them that build castles in the air; but so far substantially it worketh, not only to make a Cyrus, which had been but a particular excellency as nature might have done, but to bestow a Cyrus upon the world to make many Cyruses, if they will learn aright why and how that maker made him.“ 
ren Wirklichkeitsgrad als jeder möglicherweise tatsächlich reale Cyrus. Daß die Idee eines vollkommenen Fürsten von einem höheren Wirklichkeitsgrad ist als jeder eventuell tatsächliche, vollkommene Fürst, ist darin begründet, daß der fiktive, vollkommene Fürst als Idee und Vorbild potentiell unendlich viele vollkommene Fürsten schaffen kann, indem jeder Fürst sich dieses Ideal zum Vorbild nehmen und damit aus sich selbst einen weiteren Cyrus machen kann. Derart würde die Idee die Realität erzeugen und wäre deshalb von einem höheren Wirklichkeitsgrad.

Dichtung hat als bloße Fiktion keinen niedrigeren Realitätsgrad als ,Natur', d. h. als die tatsächliche geschöpfliche Welt, sondern hat, ganz im Gegenteil, durch ihre ,Idealität‘ einen höheren Realitätsgrad als diese. Versteht man die Stelle in dieser Form, wird deutlich, daß es Sidney um die ontologische Wertigkeit der Dichtung geht und die ganze Stelle der Versuch ist, den platonischen Vorwurf, die Dichtung sei ontologisch minderwertig, auszuhebeln. Dichtung oder Malerei, so der Vorwurf Platons im zehnten Buch der Politeia (595a-608b), sei eine bloße Abbildung der sichtbaren Wirklichkeit, die ihrerseits wiederum ein Abbild der Ideen sei. Der Tisch, den der Maler malt, entsteht nach dem Vorbild des Tisches, den der Tischler gebaut hat, der wiederum nach der Idee des Tisches entstanden ist, die der Tischler gehabt haben muß, um einen Tisch überhaupt bauen zu können. Die Idee des Tisches kommt daher, weil sie die Realität erst erzeugt, der höchste Wirklichkeitsgrad zu, während der reale Tisch nur ein Abbild dieser Idee ist, und das Bild des Tisches, das der Maler malt, nur das Abbild eines Abbildes.

Auf diesem Hintergrund wird Sidneys Argumentation verständlich. Sidney behauptet, daß die eigentliche Leistung des Dichters gar nicht in dem Werk selbst besteht (das also durchaus ontologisch minderwertig sein kann), sondern in der Idee, die dem Werk zugrundeliegt (,idea or foreconceit of the work ${ }^{\text {(626) }}$. Deswegen ist der zentrale Punkt seiner Argumentation die Behauptung, daß der Dichter auch tatsächlich eine „Idee“, d.h. die Vorstellung einer Vollkommenheit, hat, und nicht etwa einfach nur Luftschlösser entwirft, d.h. phantasiert. Die Idee einer Dichtung hat nur dann einen höheren Realitätsgrad als das Luftschloß (und als das reale Schloß), wenn sie tatsächlich Ausdruck einer Vollkommenheit, einer Idealität ist. Nur dann nämlich kann sie sich, durch ihre Modellhaftigkeit und Vorbildlichkeit, auf das reale Verhalten der Menschen auswirken. Cyrus als das von

${ }^{26}$ Die tautologische Formulierung, die den griechischen Begriff noch einmal in der Übersetzung wiederholt, steht in einer auffälligen Analogie zu Ronsards „Idées \& formes de toutes choses", vgl. oben Anm. 3. 
Xenophon formulierte, literarische Vorbild eines vollkommenen Fürsten erzeugt als Idee immer wieder reale Fürsten, die diesem Vorbild nacheifern. ${ }^{27}$

Der Vergleich der Schöpfungskraft der Natur mit der Schöpfungskraft des Dichters, so heißt es bei Sidney weiter, sei nicht vermessen, sondern ließe Gott, als dem himmlischen Schöpfer von beiden, der Natur und dem Dichter, die ihm gebührende Ehre widerfahren, denn Gott ist sowohl der Schöpfer der ,ersten“ Natur als auch der Schöpfer des Menschen, den er nach seinem eigenen Bild geschaffen und (Gen. 1.27) als Herrscher über diese ,zweite ' Natur gesetzt hat. Diese ,zweite Natur' ist die schlechte, tatsächlich vorhandene Natur, die immer nur Unvollkommenheiten hervorbringt und die der Dichter durch den beschriebenen Prozeß der Idealisierung $\mathrm{zu}$ verbessern sucht. Gott hat nicht nur eine erste, vollkommene Natur geschaffen, sondern auch noch einen Menschen, nämlich den Dichter, der, vor und über der schlechten, ,zweiten“ Natur stehend, die von Gott geschaffene, erste und vollkommene Natur in seinen Werken nachbilden kann. Gerade in dieser Schöpfungskraft des Dichters, d.h. in seiner Fähigkeit, die erste, vollkommene Natur als Idee in seinen Werken nachzubilden, besteht die Ebenbildlichkeit, nach der Gott den Menschen geschaffen hat. ${ }^{28}$

27 Es ist zurecht bemerkt worden, daß der eigentliche Anlaß von Sidneys Defence vielleicht Stephen Gossons School of Abuse (1579) sein mag, der Gegner aber, den Sidney den ganzen Traktat hindurch bekämpft, Platon ist. (Zum ersten Mal herausgestellt von Irene Samuel, „The Influence of Plato on Sir Philip Sidney's Defense of Poesy“, in: Modern Language Quarterly 1/1940, S. 383-391. Heninger, Touches of Sweet Harmony [wie Anm. 11], S. 318, Anm. 20 vermißt gerade Sidneys Auseinandersetzung mit dem platonischen Abbild-Begriff.) Eine auf Platon bezogene Interpretation des Idee-Begriffs würde die relativ weit hergeholte Annahme einer Auseinandersetzung mit Malerei-Traktaten des italienischen Manierismus überflüssig machen. Vgl. A. C. Hamilton, „Sidney's Idea of the ,Right Poet““, in: Comparative Literature 9/1957, S. 51-59, übernommen von Shepherd in seinem Kommentar zu dieser Stelle S. 157 f. und von van Dorsten S. 189 f. Hamilton und Shepherd berufen sich dabei vor allem auf Erwin Panofsky, Idea. Ein Beitrag zur Begriffsgeschichte der älteren Kunsttheorie (zuerst Leipzig 1924) und die dort behandelten Theoretiker Lomazzo und Zuccaro. Gerade was die spezifische Ausprägung des Begriffs der Idea in der Renaissance betrifft, ist Panofskys Buch jedoch scharf kritisiert worden, vgl. Wolfgang Hübener, „Idea extra artificem. Zur Revisionsbedürftigkeit von Erwin Panofskys Deutung der mittelalterlichen Kunsttheorie“, in: Lucius Grisebach/Konrad Renger (Hg.), Festschrift für Otto von Simson zum 65. Geburtstag, Berlin/Frankfurt a. M. 1977, S. 27-52. Stark von Panofsky abhängig auch Donald R. Benson, ,,Ideas“ and the Problem of Knowledge in Seventeenth Century English Aesthetics“, in: English Miscellany 19/1968, S. 83-104. Gegen einen Bezug auf den Manierismus ist Craig, Hybrid Crowth (wie Anm. 20), S. 184 ff.

28 Vgl. Sidney, Defence of Poetry (wie Anm. 20), S. 79: „Neither let it be deemed too saucy a comparison to balance the highest point of man's wit with the efficacy of nature; but rather give right honour to the heavenly Maker of that maker, who having 
$\mathrm{Da} ß$ aber der Dichter die ,erste ' Natur überhaupt nachbilden muß, ist für Sidney gerade ein Beweis für den „ersten, verfluchten Fall Adams“, durch den zwar nicht die Erkenntnisfähigkeit des Menschen, wohl aber sein Wille beschädigt wurde. Seit dem Sündenfall leidet der Mensch an der Tatsache, das Gute zwar erkennen, aber nicht mehr den Willen zu haben, es erreichen zu können. Dem gegenüber steht der Dichter, der den Menschen in seinen Werken trotzdem immer das eigentliche Gute, die Idee des Guten, vor Augen stellt und sie damit an dessen Existenz gemahnt. Die ,zweite Natur', in der der Mensch lebt, ist die schlechte, unvollkommene, durch den Sündenfall zerstörte Natur, in dieser Natur aber steht der Dichter als ein Mahnmal Gottes, das den Menschen durch seine schöpferische Kraft an die Idee und die erste, vollkommene Natur erinnert. ${ }^{29}$ Wie die ,zweite " Natur ihre unvollkommenen Geschöpfe hervorbringt, so bringt der Dichter seine vollkommenen Ideen hervor - das ist das Wesen von Sidneys Vergleich der schöpferischen Kraft von Natur und Dichter, ein Vergleich, der eben damit zur Ehre Gottes gereicht, weil es ein kleines Verdienst ist, eine Natur zu erschaffen, die unvollkommene Geschöpfe hervorbringt, aber ein großes Verdienst, einen Dichter zu erschaffen, der vollkommene Ideen hervorbringt.

Wer Sidneys Defence mit einem Verweis auf diese Stelle für das Konzept des Dichters als Schöpfers zu vereinnahmen sucht, verfälscht den Sachverhalt. Sidney vergleicht nirgends - und das in geradezu auffälliger Weise - den Dichter mit Gott, sondern immer mit der Natur. ${ }^{30}$ Das tertium

made man to His own likeness, set him beyond and over all the works of that second nature: which in nothing he showeth so much as in poetry, when with the force of a divine breath he bringeth things forth surpassing her doings - with no small arguments to the credulous of that first accursed fall of Adam, since our erected wit maketh us know what perfection is, and yet our infected will keepeth us from reaching unto it." Die obige Interpretation entspricht auch dem Verständnis Temples, der Sidneys Argument folgendermaßen paraphrasiert, Analysis (wie Anm. 20), S. 78: „Hoc loco ad refutandam prolepsin digrederis. Objici enim potest naturae opus reipsa existere: opus vero poetae figmento cogitationis: ideoque naturam a poeta non vinci dignitate. refellitur prolepsis dum subjectum illud notas, in quo praestantia artificis \& dignitas posita est. Posita est (inquis) in idea operis non in ipso opere."

29 Damit ist die Existenz des Dichters ein Beweis für den Sündenfall Adams. Daß der Dichter in seinen Werken die erste Natur wiederauferstehen lassen kann, ist ein Argument dafür, daß es diese erste Natur gegeben hat. Sidneys Argument richtet sich also nicht an die Gläubigen (credulous), sondern an die Ungläubigen (incredulous). Nach dem kritischen Apparat von van Dorsten existieren in den Drucken und Handschriften der Defence beide Varianten, van Dorsten hat sich jedoch für „credulous“" entschieden, vgl. die vorherige Anm.

30 Die Stelle muß als explizite Auseinandersetzung mit Scaliger gelesen werden, worauf bereits oft hingewiesen worden ist, merkwürdigerweise ohne die Unterschiede zu vermerken. Auch Scaliger vergleicht Poetices libri septem I.1 (wie Anm. 22), S. $71 \mathrm{f}$. 
comparationis seines Vergleiches ist die Schöpfungskraft, die als gefallene Natur nur Unvollkommenheiten und ,gemischte Charaktere" hervorbringt, allein im Dichter aber Ideen und Ideale. Die dichterische Schöpfungskraft, wie Sidney sie konzipiert, ist damit das im Vergleich zur Natur genau umgekehrte Prinzip, und der Dichter ist kein Gott, der eine Welt aus seiner Phantasie heraus erschafft, wie es die Romantik wollte, sondern er ist, genauso wie die Natur, von Gott geschaffen. Nicht eine Welt entwirft der Dichter, die allein den Gesetzen seiner Phantasie gehorcht, sondern er rekonstruiert das Ideal einer Welt vor dem Sündenfall.

Sidneys Umdeutung des platonischen Begriffes der Idee ${ }^{31}$ und damit die ontologische Aufwertung der dichterischen Fiktion findet ihre Ergänzung in dem Versuch, den ontologischen Begriff der Idee (aus der platonischen Tradition) mit dem logischen Konzept des exemplum (aus der aristotelischen Tradition) zu verschmelzen. Nachdem Sidney festgestellt hat, daß seine bisherigen, aus der Etymologie abgeleiteten Argumente wohl von den wenigsten verstanden würden, heißt es weiter, er werde nun in einem zweiten, „gewöhnlicheren“ Ansatz (,,a more ordinary opening“) versuchen, die Richtigkeit der aufgestellten Behauptungen aus der Beschreibung des Wesens der Dichtung ableiten. ${ }^{32}$

Sidney entwickelt also in dem folgenden Teil der Defence nicht eine zweite Definition der Dichtkunst (wie gelegentlich behauptet worden ist), sondern - und darin folgt er dem von der Rhetorik und Dialektik vorgegebenen Aufbauschema - er nähert sich seinem Gegenstand zuerst mit Argu-

die Dichtung mit den anderen Wissenschaften und Künsten und sieht den Unterschied darin, daß dort, wo der Dichter eine ,andere Natur“ hervorbringt, die anderen Wissenschaften nur „Darsteller“ oder „Schauspieler“ (,actores“, wie bei Sidney) der Natur sind. Scaliger bildet dann die Analogie allerdings zwischen Gott und Natur auf der einen und Dichter (,zweiter Gott“) und ,zweiter Natur“ auf der anderen Seite (wie auch Puttenham, vgl. oben Anm. 12). Sidneys Modifikation der Analogie mag auf seinen puritanischen Hintergrund zurückgehen. Sidney setzt sich allerdings auch bei der Definition des Dichters scharf von Scaliger ab, denn wo Scaliger den Begriff des Dichters als Schöpfer auf das Bilden von Versen zurückführt (und folglich auch die Dichtung über ihre Versform definiert), bezieht Sidney den Begriff des Schöpfers auf das Erfinden von Fiktionen und definiert die Dichtung über ihren fiktionalen Charakter.

31 Am nähesten kommt Sidneys Begriff der Idee Castigliones Libro del cortegiano (1528), worauf Annabel M. Patterson, „Tasso and Neo-Platonism. The Growth of his Epic Theory“, in: Studies in the Renaissance 18/1971, S. 105-133, hier S. $121 \mathrm{f}$. hingewiesen hat. In seinem Vorwort erklärt Castiglione, mit seinem Buch die Idee eines Hofmanns formulieren zu wollen, in dem Sinne, in dem Platon das Ideal eines Staates, Cicero das Ideal eines Redners und Xenophon das Ideal eines Fürsten aufstellen wollte. Insbesondere die Cyrus-Parallele ist auffällig.

32 Vgl. Sidney, Defence of Poetry (wie Anm. 20), S. 79, Z. 31-34. 
menten, die aus äußerlichen Topoi (Alter der Dichtkunst, Etymologie der Benennungen vates und poeta) abgeleitet sind, und kommt dann zu den Topoi, deren Argumente das Wesen eines Gegenstandes bestimmen und aus denen sich deshalb seine Definition und Beschreibung zusammensetzt: die Gattung, zu der er gehört; die ,spezifische Differenz', die ihn von den anderen Arten dieser Gattung unterscheidet; die „distributio“ oder „divisio“, d. h. die Aufgliederung der Arten und Teile, aus denen er sich zusammensetzt; seine Eigenschaften; seine Ursachen; seine Folgen usw. ${ }^{33}$ Die confirmatio (die positive Bestimmung des Gegenstandes, der später der refutatio als Widerlegung möglicher Einwände folgt) der Defence entwickelt deshalb schon aus Gründen ihrer rhetorischen Struktur nicht zwei Definitionen der Dichtung, sondern Sidney beginnt seine Verteidigung der Dichtung mit aus dem Topos der Etymologie abgeleiteten Argumenten und stößt dabei auf

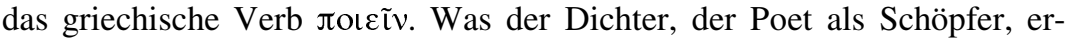
schafft, ist eine ,andere Natur“, und genau an dieser Stelle muß Sidney sich mit dem platonischen Vorwurf der ontologischen Minderwertigkeit der Dichtung konfrontiert sehen. Was folgt, ist seine Umwertung des Abbildcharakters der Dichtung in den Begriff der Idee. Was Sidney bis zu diesem Punkt gegeben hat, sind aus der Etymologie abgeleitete Argumente.

Die eigentliche, erste und einzige Definition der Dichtung folgt erst jetzt, wenn Sidney die Dichtung als Nachahmung mit dem Zweck der Information und Unterhaltung (docere und delectare) definiert. ${ }^{34}$ Sidney folgt weiterhin den methodischen Vorgaben der Rhetorik, wenn er zuerst die verschiedenen Arten (göttlich inspirierte, didaktische und ,eigentliche‘ Dichtung)

33 Dieses Vorgehen wird in der melanchthonisch oder ramistisch geprägten Rhetorik und Dialektik der Zeit als Methode bezeichnet. Vgl. dazu die Bemerkungen von Webster in seiner Einleitung zu Temple, Analysis, sowie ausführlicher in Webster, Oration and Method (wie Anm. 20). Temple selbst beschreibt das Verfahren Sidneys Analysis (wie Anm. 20), S. 80, in diesem Sinne: „Adhuc Poesin ornasti ab adjuncto triplici, nimirum ab antiquitate, communitate, nominibus. Sequitur poeticae facultatis laus primum e definitione, tum e Distributione." Diese zeitgenössische rhetorische Analysen unterscheidet sich damit stark von modernen rhetorischen Analysen, die von antiken Modellen ausgehen und die Defence als eine Rede des genus iudiciale analysieren, vgl. etwa Kenneth Myrick, Sir Philip Sidney as a Literary Craftsman, Lincoln ${ }^{2} 1965$ (1935), S. 46-83, oder Shepherds Einleitung zu Sidney: Apology for Poetry (wie Anm. 20), S. $13 \mathrm{f}$. Beide Modelle müssen sich nicht ausschließen, es ist jedoch offensichtlich, daß die zeitgenössischen Analysen erstens von einem anderen Begriff von Rhetorik ausgehen und zweitens eine ungleich höhere Detailschärfe besitzen.

34 Sidney, Defence of Poetry (wie Anm. 20, S. $79 \mathrm{f} .:$ „Poesy therefore is an art of imitation, for so Aristotle termeth it in the word $\mu$ í $\mu \varepsilon \sigma 1 \varsigma$ - that is to say, a representing, counterfeiting, or figuring forth - to speak metaphorically, a speaking picture - with this end, to teach and delight." 
und Teile (die poetischen Gattungen und Formen) der Dichtung bestimmt und sich dann der Dichtung selbst durch die Bestimmung ihres Gattungsbegriffes (genus) nähert. Dieses genus der Dichtung, als imitatio, ist der Begriff des Lernens im weitesten Sinne ${ }^{35}$. Innerhalb des menschlichen Wissens verortet Sidney die Dichtung am Schnittpunkt von Philosophie und Historie, d.h. genauer, zwischen der Unterrichtung über die Ursachen und Folgen menschlichen Handelns, wie es die Moralphilosophie in abstrakter, begrifflicher Form lehrt, und der ,exemplarischen', konkreten Darstellung solchen Handelns, wie es sich in der Historie findet.

Dichtung hat denselben Zweck wie die Moralphilosophie, nur daß dort, wo diese sich der logischen Form des Syllogismus bedient und moralische Vorschriften deduktiv ableitet, die Dichtung sich der Form des exemplum bedient und diese moralischen Vorschriften am Einzelfall illustriert. Die Form des exemplum hat die Dichtung mit der Historie gemeinsam, im Unterschied zu dieser allerdings ist sie nicht an das Partikuläre und Individuelle der tatsächlichen Geschichte gebunden, sondern kann ein solches exemplum gemäß den Erfordernissen der moralischen Regel, die sie illustriert, erfinden. Das exemplum entspricht deshalb der poetischen Form der Parabel oder des Gleichnisses. Diese Form erfüllt den Zweck des docere durch die moralphilosophische Lehre, die ihr zugrundeliegt, den Zweck des delectare durch die anschauliche, narrative Form, die sie mit der Historie gemeinsam hat, und darüber hinaus noch den Zweck des movere, indem sie Affekte erregen kann, was der bloßen moralphilosophischen Regel nicht gelingen kann. Sidney illustriert diese Form in seiner Defence, auch hierin ganz traditionell, unter anderem mit den neutestamentlichen Gleichnissen, etwa dem Gleichnis vom verlorenen Sohn, den Gleichnissen Nathans, den Fabeln Äsops und der Fabel des Menenius Agrippa, mit der dieser den Plebs Roms in die Stadt zurückholte. ${ }^{36}$

Durch die Form des Gleichnisses vereinigt die Dichtung die Vorteile von Moralphilosophie und Historie. Der Dichter illustriert in einem ,feigned example $^{637}$, eben einem Gleichnis, die philosophische Regel und hat deshalb dem Philosophen die Anschaulichkeit des Bildes voraus, die eine viel größere Überzeugungskraft hat als die ,wortreiche Beschreibung“ (,wordish description“) des Philosophen. Zwar erfüllen die gelehrten philosophischen Definitionen das menschliche Gedächtnis mit den untrüglichen Grundlagen allen Sachverstandes, doch gerade für das Vorstellungs- und Urteilsvermögen bleiben diese Grundlagen dunkel, solange sie nicht durch das ,spre-

\footnotetext{
Vgl. S. 82.

Vgl. S. $86 \mathrm{ff}$.

S. 89.
} 
chende Bild“ (,speaking picture“) der Dichtung veranschaulicht und vorgestellt werden. ${ }^{38}$

Dem Historiker dagegen hat der Dichter gerade die Fiktionalität seiner Exempel voraus. ${ }^{39}$ Ein Bild, das Vespasian so darstellt, wie er wirklich aussah, ist, wenn es um die Frage der historischen Wahrhaftigkeit geht, einer frei erfundenen Darstellung vorzuziehen. Wenn es dagegen um den moralpädagogischen Nutzen geht, den man aus einem exemplum ziehen kann, ist der erfundene Cyrus Xenophons dem historisch korrekten Cyrus Justins vorzuziehen.$^{40}$ Der Dichter übertrifft den Historiker genau in so weit, als er sein exemplum nach dem bilden kann, was am vernünftigsten ist, wo hingegen der Historiker auf das bloße , so war es' verwiesen ist und deshalb oft genug darstellen muß, wie der sogenannte Zufall die sachverständigsten und vernünftigsten Vorhaben zunichte gemacht hat. Aus dieser Tatsache aber folgt kein moralpädagogischer Nutzen, sondern höchstens Resignation. ${ }^{41}$

Daß Sidney sich hier wieder der Cyropaideia Xenophons als Beispiel bedient, ist kein Zufall. Beide Modelle, Idee und exemplum, funktionieren so, wie Sidney sie beschreibt, analog. Was in dem ,gewöhnlicheren“ rhetorisch-dialektischen Ansatz ein exemplum heißt, hat Sidney zuvor in der „ungewöhnlichen“ Sprache Platons als die „Idee“ einer Dichtung eingeführt. Indem Sidney das poetische exemplum von der moralischen Indifferenz des historischen exemplum befreit und es auf moralische Vorbildlichkeit festlegt, kann es zu der "Idee“ werden, die, in Sidneys Umdeutung der platonischen Hierarchie, die Dichtung von ihrer ontologischen Minderwertigkeit befreit. Das „Vorbringen“ der Idee in einer Dichtung entspricht dem „Vor-Augen-Stellen“ der philosophischen Regel und des moralischen Vorbildes im erfundenen exemplum. ${ }^{42}$

38 Vgl. S. 86.

39 Für dieses Argument beruft Sidney sich zu Unrecht auf das neunte Kapitel der aristotelischen Poetik, denn Aristoteles begründet 1451b die Überlegenheit der Dichtung mit ihrer größeren Allgemeingültigkeit, nicht mit ihrer moralischen Vorbildlichkeit.

40 Vgl. Sidney, Defence of Poetry, S. 88.

${ }^{41}$ Vgl. S. 89: ,,[... the poet doth so far exceed him as he is to frame his example to that which is most reasonable (be it in warlike, politic, or private matters), where the historian in his bare Was hath many times that which we call fortune to overrule the best wisdom." Auch damit widerspricht Sidney Aristoteles, der Poetik 1460b drei Möglichkeiten der dichterischen oder malerischen Nachahmung unterscheidet: die Nachahmung des Zustandes, in dem die Dinge sind, in dem sie zu sein scheinen oder in dem sie sein sollten.

42 So auch das Verständnis Temples, der Analysis (wie Anm. 20), S. 100, das exemplum in dieser Funktion mit ,perfecta imago“" paraphrasiert. 
Wenn Sidney die Dichtung als imitatio definiert und sich dafür auf Aristoteles beruft, modifiziert er also den aristotelischen Begriff in demselben Maße wie zuvor den platonischen Begriff. Die imitatio der Dichtung ist nicht die Nachahmung einer Handlung, wie bei Aristoteles, sondern die Darstellung einer Idee, d.h. das Vorstellen und Vor-Augen-Stellen eines Ideals im , sprechenden Bild ' der Dichtung, wobei der Effekt des docere von dem Ideal und der Effekt des delectare von der Vorstellung und narrativen Darstellung ausgeht. Die Nachahmung der Dichtung ist die Illustration eines Ideals. Und wie der Dichter von diesem Ideal ausgeht und es dann in einem exemplum oder Gleichnis illustriert, so geht umgekehrt der Leser von dieser Illustration aus und schließt von dort aus auf das zugrundeliegende Ideal zurück. Die erzählerische Gestalt einer Dichtung (,narration“) ist nichts anderes als der anschaulich dargestellte und phantasievoll ausgestaltete Entwurf (,imaginative ground-plot“), wie er der dichterischen Fiktion als moralisch nützliches Argument (,profitable invention“) zugrundeliegt. Diese Fiktion kann deshalb allegorisch oder figurativ heißen. ${ }^{43}$

Weil der Leser weiß, daß es sich bei der dichterischen Darstellung um eine Fiktion handelt, weiß er auch, daß diese Fiktion in ihrem Wortsinn allegorisch zu verstehen ist, in ihrem metaphorischen Sinn aber Ausdruck einer Wahrheit, eines Ideals ist. Der Leser bedient sich der dichterischen ,narratio“ nur, um von ihr auf die gleichnishaft darin verborgene Wahrheit zurückzuschließen. Dichtung ist deshalb keine Lüge, so lautet Sidneys Entkräftung des platonischen Vorwurfes, denn daß die bloß fiktionale Darstellung als solche wahr ist, wie die Erzählung des Historikers wahr ist, hat der Dichter nie behauptet. Dichter lügen nicht, weil sie das, was sie im wörtlichen Sinne sagen, gar nicht als wahr behaupten. ${ }^{44}$ Damit bekommt die Fiktionalität der Dichtung mit dem Begriff der Idee nicht nur einen ontologisch, sondern - mit der Gleichnishaftigkeit - auch einen logisch unabhängigen Charakter zugesprochen.

43 Sidney, Defence of Poetry (wie Anm. 20), S. 103: „If then a man can arrive to that child's age to know that the poets' persons and doings are but pictures what should be, and not stories what have been, they will never give the lie to things not affirmatively but allegorically and figuratively written. And therefore, as in history, looking for truth, they may go away full fraught with falsehood, so in poesy, looking but for fiction, they shall use the narration but as an imaginative ground-plot of a profitable invention."

44 S. 102: „Now, for the poet, he nothing affirms, and therefore never lieth. For, as I take it, to lie is to affirm that to be true which is false." 


\section{William Temples Analysis tractationis de Poesi}

Sidney selbst hat in diesem letzten Punkt schärfste Kritik erfahren, und zwar gerade von seinem eigenen Sekretär, William Temple, der in seiner Analysis tractationis de Poesi contextae a nobilissimo viro Philippe Sidneio equite aurato (wahrscheinlich zeitgleich mit der Defence selbst entstanden) die Argumentationsstruktur der Defence nach der ramistischen Methode analysiert und in zentralen Punkten widerlegt. ${ }^{45}$

Temples Kritik setzt an Sidneys Definition der Lüge an. Stark verkürzt lautet seine Argumentation: Wer lügt, bestreitet, daß etwas wahr ist, was tatsächlich wahr ist. Um den Tatbestand der Lüge zu erfüllen, muß er nicht eigens noch behaupten, daß seine Lüge wahr ist, sondern die eigentliche falsche, lügnerische Aussage genügt völlig. Anders gesagt: Wer die Aussage macht: „Der Mensch ist kein Lebewesen“ muß dieser Aussage nicht eigens noch hinzufügen, daß er sie behauptet, sondern indem er sie macht, sagt er etwas Falsches, d. h. er lügt. Der Tatbestand der Lüge besteht nicht in einem mentalen Akt der Affirmation einer Behauptung, sondern in der Behauptung selbst, weshalb das Wesen der Fiktion zutreffend als Lüge bezeichnet werden kann. ${ }^{46}$

Ebenso scharf ist Temples Kritik an Sidneys Definition der Dichtung, die er folgendermaßen reformuliert: „Dichtung ist die Kunst der Nachahmung oder Erfindung [fictio], mit dem Zweck der Information [docere] und Unterhaltung [delectare]. ${ }^{“ 47}$ Das Gegenargument Temples lautet:

Dies ist jene Definition, verehrtester Philipp, die den gesamten Streitpunkt enthält, und die gleichsam das Fundament ist, auf dem die von dir darauf errichtete Abhandlung über die Dichtung fast ausschließlich beruht. Laß uns deshalb sehen, ob sie das Wesen der definierten Sache richtig erläutert und definiert. Du willst, daß das Wesen der Dichtung als eine Art von Erfindung [fictio] verstanden wird. Was aber ist diese Erfindung anderes als die inventio eines Sachverhaltes, der noch nicht existiert? Wer etwas erfindet, der erfindet logische Argumente, nämlich Ursachen, Wirkungen, Subjekte, Adjuncta, Gegensätzliches und all das übrige, das aus diesen hervorgeht. Wenn Ovid den Palast der Sonne erfindet, erfindet er auf diese Art die Wirkursache, aus der der Palast gebaut wird, das Material, aus dem er gebaut wird, und die Adjuncta, mit denen er geschmückt wird. Ursachen, Wirkungen, Subjekte, Adjuncta und die übrigen Argumente zu erfinden [fingere] ist aber nichts anderes, als Ursachen, Wirkungen, Subjekte und Adjuncta zu finden [inve-

45 Bibliographische Angaben vgl. oben Anm. 20.

46 Vgl. Temple, Analysis (wie Anm. 20), S. 136.

47 S. 80: „Poesis est ars imitationis seu fictionis ad Docendum \& delectandum.“ Übers. hier und im folgenden vom Verfasser. Der Begriff der Fiktion taucht bei Sidney an dieser Stelle zwar nicht auf, dennoch wird man Temple wohl zustimmen müssen, daß er das Wesen der imitatio, wie Sidney sie bestimmt, am besten wiedergibt. 
nire]. Deswegen wird also die Erfindung dasselbe sein wie die inventio eines Sachverhaltes, der noch nicht existiert. Wenn dies aber so ist, dann wird die Kunst der Erfindung nicht zur Dichtung gehören, sondern zur dialektischen inventio, durch die eben nicht nur wahrhafte Sachverhalte erdacht werden können, sondern auch fiktive. Ich gebe zu, daß die Sachverhalte selbst, die erfunden werden, zu einer anderen Disziplin gehören, zur Ethik meistens oder zur Physik, genauso wie jene Argumente, die im Wesen der Sachverhalte erkannt werden [im Gegensatz also zu den erfundenen] und dort von Bedeutung sind. Aber die Erfindung selbst ebenso wie das Erdenken dieser Argumente ist die Tätigkeit einer angeborenen oder ,künstlich“ angelernten Methode des Findens. ${ }^{48}$

Temple argumentiert auf dem Hintergrund der zeitgenössischen Definitionstheorie. Wenn die Erfindung das Wesen der Dichtung ausmachen soll, muß diese Erfindung allein der Dichtung eigentümlich sein. Dazu aber müßte sich die fictio, die Erfindung der Poetik, wesenhaft von der inventio, der Findung von Argumenten, wie sie zur Dialektik gehört, unterscheiden. Temple stellt dagegen gerade die Behauptung auf, daß die fictio nichts anderes als die inventio eines noch nicht existierenden Sachverhaltes und also nur eine spezifische Ausprägung der dialektischen inventio ist. Was der Dichter erfindet, sind, im logischen Sinne, Argumente. Wenn Ovid in den Metamorphosen den Palast der Sonne erfindet, findet er Argumente aus den dialektischen Topoi der Ursachen, Wirkungen, Subjekte und Adjuncta, indem er z. B. ein Motiv erfindet, warum dieser Palast erbaut wird, bedient er sich des Topos der causa efficiens, der Wirkursache. Indem er ein Material erfindet, aus dem dieser Palast gebaut wird, bedient er sich des Topos der materialen Ursache, indem er die architektonische Gestaltung dieses Palastes erfindet, bedient er sich des Topos der Adjuncta, usw. Die Tätigkeit der dichterischen Erfindung ist die Anwendung der dialektischen inventio. Der

48 Ebd.: „Haec est illa definitio (illustrissime Philippe) quae totam controversiam continet: \& qua tanquam fundamento instituta a te de Poesi tractatio fere nititur. Videamus igitur rectene rei definitae naturam explicet \& definiat. Vis tu poeseos naturam fictione quadam comprehendi. Ecquid fictio ista aliud est quam inventio rei quae nondum extitit? Qui fingit, is logica argumenta fingit, nempe causas, effecta, subjecta, adjuncta, dissentanea, comparata aut caetera quae ex istis oriuntur. Sic Ovidius fingens regiam Solis finxit efficientem causam a qua constructa est, materiam ex qua conflata est, adjuncta quibus ornata est. At fingere Causas, effecta, subjecta, adjuncta, caeteraque argumenta, nihil aliud est quam invenire causas, effecta, subjecta, adjuncta. Quamobrem fictio erit idem quod rei, quae nondum extiterit, inventio. Id si ita est, ars fictionis non ad poesin sed ad dialecticam inventionem pertinebit: qua non solum res verae sed etiam fictitiae cogitantur. Fateor res ipsas quae finguntur, esse alterius disciplinae, ethicae quidem plerunque aut physicae, non minus quam argumenta illa quae in rerum natura cernuntur et vigent. At ipsa fictio perinde ac excogitatio horum argumentorum est actio nativae vel artificiosae rationis in inveniendo.“ 
,Stoff ‘ des Dichters, in moderner Terminologie, ist ein Konglomerat von Argumenten im dialektischen Sinne. Wenn dies aber so ist, unterscheidet sich die Erfindung nicht wesenhaft von der inventio der Dialektik und kann also auch nicht das Definiens der Dichtung sein.

Der Dichter bedient sich bei seiner Tätigkeit des Erfindens der dialektischen inventio, sei es in der Form, in der jedem Menschen diese Fähigkeit in einem bestimmten Maße angeboren ist, sei es in der Form, in der sich jemand methodisch durch die Dialektik in dieser Fähigkeit ausgebildet hat. Damit ist natürlich auch gesagt, daß die Tätigkeit des Erfindens keine spezifisch dichterische Fähigkeit ist, sondern in der Tat jeder, der tagtäglich Argumente für oder gegen etwas findet, sich dieser Tätigkeit bedient. Die dichterische Fähigkeit, Fiktionen zu bilden, wie Sidney sie an „Heroen, Halbgöttern, Zyklopen und Chimären" illustriert, ist als Fähigkeit keine spezifisch dichterische, denn ,diese Erfindung wird nicht durch die Kraft eines dichterischen Vermögens gebildet“ ".

Die Poetik als Disziplin steht bei Temple als Schwesterdisziplin von Grammatik, Dialektik und Rhetorik wieder an dem traditionellen Ort, den sie innerhalb des humanistischen Triviums einnimmt. Der ramistischen Tradition entsprechend definiert Temple die Dichtung über den Vers. ${ }^{50}$ Während die Aufgabe der Dialektik der inventive und argumentative Teil der Kommunikation und die Aufgabe der Rhetorik die sprachliche Gestaltung (elocutio) ist, besteht die Aufgabe der Poetik nur in der metrischen Form und einer spezifisch poetischen Ausprägung der rhetorischen elocutio (d.h. vor allem einem freizügigeren Gebrauch der rhetorischen Figuren). Temple unterscheidet sich mit dieser Aufgliederung nicht wesentlich etwa von der Position, die über hundert Jahre zuvor schon Rudolf Agricola in seinen De inventione dialectica libri tres (1479, Druck 1539) formuliert und Petrus Ramus zum Unterscheidungsprinzip von Rhetorik, Dialektik und Poetik erklärt hatte. Im Gegensatz zu Puttenham und Sidney argumentiert Temple damit von einer traditionellen Definition der Dichtung ausgehend, die diese in ihrem schulisch-akademischen Rahmen verweist und das dichterische Schaffen nicht von dem unterscheidet, was in der rhetorischen oder dialektischen inventio geschieht. Auch der Dichter bleibt damit natürlich auf den schulisch-akademischen Rahmen verwiesen.

49 S. 78: ,[...] fictionem istam non effici vi poeticae facultatis.“

50 Vgl. S. 88 . 


\section{Schlußfolgerungen}

Murray W. Bundy hat die seitdem gelegentlich wiederholte These formuliert, die Renaissance habe den rhetorischen Term der inventio neu im Sinne der imaginatio definiert und damit einen entscheidenden Schritt hin zum Begriff einer kreativen Phantasie getan. ${ }^{51}$ Dem gegenüber habe ich zu zeigen versucht, daß das Problem der Poetiken nicht ist, eine Verbindung zwischen imaginatio und Dichtung herzustellen, sondern gerade umgekehrt, diese gefährliche Verbindung so zu erklären, daß sie dem Rang der Dichtung keinen Abbruch tut. Damit sind auch zwei unausgesprochene Annahmen, die der These Bundys zugrundeliegen, angegriffen. Die erste dieser Annahmen lautet, daß die Poetik der Renaissance teleologisch als eine Entwicklung weg von der Rhetorik, hin zur ,kreativen Phantasie' des 18. Jahrhunderts zu verstehen ist. Das Ziel meiner Interpretation war dagegen, die Poetik der Renaissance aus dem Kontext ihrer Zeit heraus, und das heißt, in Abgrenzung von einem schulischen Modell von Dichtung einerseits und in Anschluß an die psychologisch-medizinischen Vorstellungen andererseits, verständlich zu machen. Die zweite dieser Annahmen Bundys lautet, daß die rhetorische inventio kein ,kreatives Potential ' besitze. Wie die Analysis von Temple jedoch zeigt, galt die rhetorische inventio sehr wohl als eine produktive Technik. Der entscheidende Unterschied besteht nicht zwischen einer mechanischen inventio und einer kreativen Phantasie, sondern in der Tatsache, daß die inventio, im Gegensatz zur imaginatio, Argumente erzeugt, und nicht Vorstellungsbilder.

Vor dem Hintergrund von Temples Analysis und ihrer traditionellen Interpretation des dichterischen Schaffens zeigt sich, daß das Bemühen von Puttenham und Sidney jedoch gar nicht diesem Gegensatz gilt, sondern der Aufwertung von Dichter und Dichtung. Die Begriffe der imaginatio, der „Schöpfung“ und der „Idee“, deren Puttenham und Sidney sich, ausgehend von der neuplatonischen Dichtungstheorie, zu diesem Zweck bedienen, zwingen sie jedoch an anderer Stelle in eine defensive Position, denn über diese Begriffe gerät der Dichter in die Nähe des Verrückten und des phantasierenden Fieberkranken. Puttenham konnte zwar das dichterische Schaffen qua imaginatio zu einer „Schöpfung“ erklären, mußte aber die Kontrolle der imaginatio durch die ratio scharf hervorheben. Sidney konnte den Gegenstand der Dichtung zwar zu einer Idee erklären und damit für die Dichtung einen ganz anderen Rang als den einer bloß propädeutischen Hilfsdisziplin beanspruchen, mußte diese Idee andererseits aber mit dem rhetorischen exemplum identifizieren, um nicht den Verdacht aufkommen zu lassen, diese

51 Vgl. Bundy, ,Invention ‘ and,Imagination' (wie Anm. 11). 
Idee sei ein bloßes „Luftschloß“, auf demselben logischen und ontologischen Realitätsgrad wie die Wahnvorstellung eines Verrückten.

Temple dagegen widerlegt die Argumentation Sidneys, indem er sie auf das traditionelle, humanistische Modell reduziert: das dichterische Schaffen ist eine Form der logischen inventio, in deren Verlauf Argumente gefunden werden, die der Dichter dann in der rhetorischen elocutio sprachlich ausgestaltet und nach Anleitung der Poetik in Verse bringt. Innerhalb dieses technischen Verfahrens erscheint der Dichter freilich weniger als höfisches Rollenmodell denn als Anwender von Techniken, die jeder Student in seiner universitären Vorschule (nämlich Grammatik, Dialektik und Rhetorik als den Fächern des Triviums) vermittelt bekommt. Für eine Poetik als solche, das heißt für eine Dichtungslehre, geschweige denn für eine emphatische „Verteidigung der Dichtung“, ist in diesem System eigentlich kein Platz, denn Poetik heißt hier nicht viel mehr als Metrik und Gattungslehre. Allerdings kommt ein solcher Begriff von Dichtung auch nicht in die Nähe der Verrücktheit, wie sie, um darauf abschließend noch einmal hinzuweisen, Theseus im Midsummer Night's Dream dem Dichter zum Vorwurf macht, wenn es heißt: „The lunatic, the lover, and the poet/ Are of imagination all compact“ ${ }^{52}$

52 wie Anm. 1. 